\title{
Developing smarter host mixtures to control plant disease
}

Article

Accepted Version

Mikaberidze, A., McDonald, B. A. and Bonhoeffer, S. (2015) Developing smarter host mixtures to control plant disease. Plant Pathology, 64 (4). pp. 996-1004. ISSN 00320862 doi: https://doi.org/10.1111/ppa.12321 Available at https://centaur.reading.ac.uk/86109/

It is advisable to refer to the publisher's version if you intend to cite from the work. See Guidance on citing.

To link to this article DOI: http://dx.doi.org/10.1111/ppa.12321

Publisher: Wiley

All outputs in CentAUR are protected by Intellectual Property Rights law, including copyright law. Copyright and IPR is retained by the creators or other copyright holders. Terms and conditions for use of this material are defined in the End User Agreement.

\section{www.reading.ac.uk/centaur}

\section{CentAUR}

Central Archive at the University of Reading

Reading's research outputs online 


\title{
How to develop smarter host mixtures to control plant disease?
}

\author{
Alexey Mikaberidze, \\ Bruce A. McDonald, \\ Sebastian Bonhoeffer
}

Running Head: Smarter host mixtures

Affiliation: Institute of Integrative Biology, ETH Zurich

Keywords: epidemiology, plant disease, mathematical model, host-pathogen interaction, host diversity, cultivar mixture, host mixture, multiline cultivar, population dynamics

*alexey.mikaberidze@env.ethz.ch 
2 Adaptation of plant pathogens to disease control measures (both chemical and genetic) is facilitated 3 by the genetic uniformity underlying modern agroecosystems. One path to sustainable disease 4 control lies through increasing genetic diversity at the field scale by using genetically diverse host mixtures. We utilized a robust population dynamical approach to investigate how host mixtures can improve disease control. We find that when pathogens exhibit host specialization, the overall disease severity decreases with the number of components in the mixture. This finding makes it possible to determine an optimal number of components to use in the host mixture. In a simple case where two host varieties are exposed to two host-specialized pathogen species or strains we identify quantitative criteria for optimal mixing ratios. Using these model outcomes, we propose 11 ways to optimize the use of host mixtures to decrease disease in agroecosystems. 


\section{Introduction}

The two most widely used disease control measures are applications of chemicals (fungicides and antibiotics) and breeding for disease resistant crop cultivars by incorporating resistance genes. Both of these control measures are highly vulnerable to pathogen adaptation. Many pathogens have repeatedly evolved to overcome resistance conferred by major resistance genes (reviewed in (McDonald and Linde, 2002; Parlevliet, 2002; Singh et al., 2011)). Similarly, many fungicides rapidly lose their efficacy because of the emergence and fixation of mutations encoding fungicide resistance (e.g. (Torriani et al., 2009; Brunner et al., 2008). As a result of pathogen evolution, the current commonly practiced disease control measures will likely be inadequate to enable a sustainable intensification of food production.

Quantitative or partial resistance is thought to be more durable (Parlevliet, 2002; Papaïx et al., 2011), but has not been as widely utilized as major gene resistance. Recent research has begun to provide insights into the molecular mechanisms responsible for quantitative resistance (Poland et al., 2009; Kou and Wang, 2010), but studies that include quantitative resistance in epidemiological models are rare (Lo Iacono et al., 2012). Pathogens can still adapt to quantitative resistance leading to an erosion of its effects (Stuthman et al., 2007; Mundt et al., 2002; McDonald and Linde, 2002; Lehman and Shaner, 1997), although at a much slower pace compared to major resistance genes.

More effective and longer-lasting disease control methods are urgently needed to achieve a sustainable intensification of crop production. One way to develop such methods is to focus on the underlying properties of modern agricultural ecosystems (agroecosystems) that make them vulnerable to plant pathogens. Compared to natural ecosystems, agroecosystems are more environmentally homogeneous, have a higher density of plants, and possess much less genetic and species 
diversity. It is increasingly recognized that these underlying properties of agroecosystems, especially the lack of genetic diversity due to the dominance of monoculture crops grown as clones, make them especially susceptible to disease epidemics (Mundt, 2002; Wolfe, 2000; Garrett and Mundt, 1999).

For these reasons, many researchers propose to deliberately increase genetic diversity in agroecosystems (McDonald, 2014; Newton et al., 2009; Zhu et al., 2000) in order to decrease disease in the short-term and enhance the durability of disease resistance in the long-term. This diversity can be created within a single genetic background by developing multiline cultivars (Browning and Frey, 1969) or involve many genetic backgrounds by using variety mixtures (Wolfe, 1985; Smithson and Lenne, 1996; Mundt, 2002). In this study, we do not distinguish between multiline cultivars and variety mixtures and we will refer to both options simply as host mixtures.

Many field experiments have been performed to determine whether host mixtures reduce the amount of fungal disease on crop plants (e.g. (Huang et al., 2012; Ning et al., 2012; Newton and Guy, 2011; Cowger and Mundt, 2002; Zhu et al., 2000; Newton et al., 1997; Mundt et al., 1994; Chin and Wolfe, 1984), see also reviews (Walters et al., 2012; Mundt, 2002; Finckh et al., 2000; Smithson and Lenne, 1996; Wolfe, 1985) and references therein). The findings of over 30 studies (mostly in barley, wheat, rice and beans) were summarized in (Smithson and Lenne, 1996). The vast majority of experiments showed less disease in mixtures as compared to the mean of the pure stands for obligate pathogens such as rusts and mildews. However, there was a large variation in the percentage of disease reduction: for example, between $9 \%$ and $80 \%$ for powdery mildew in barley, and between $13 \%$ and $97 \%$ for stripe rust in wheat. A recent meta-analysis of stripe rust on wheat considered 161 mixture cases reported in 11 publications (Huang et al., 2012). In $83 \%$ of these cases the average disease level was found to be lower in mixtures compared to the mean of the pure stands. A reduction in disease of between $30 \%$ and $50 \%$ was found most frequently. 
A large-scale study performed in China demonstrated that row mixtures of rice varieties could strongly reduce rice blast (Zhu et al., 2000). Thus, host mixtures reduce the amount of disease in most studied cases, but the outcomes exhibit a wide variation, even within a single study (for example (Cowger and Mundt, 2002)).

This variation is one of the reasons why multilines and cultivar mixtures have so far gained little acceptance among seed companies or growers. To achieve reliable disease control, we need to identify the conditions under which mixtures work best and use this knowledge to design optimal mixtures. This requires a better understanding of the underlying mechanisms of disease reduction in mixtures. Our study contributes to this understanding in three important ways by using a population dynamics model of plant-pathogen interactions. First, we identified conditions where mixtures are superior compared to pure stands. Second, we defined optimal ratios of components to include in the mixture. Third, we determined optimal numbers of components to include in the mixture.

This was done by exploring possible disease outcomes when two or more hosts are mixed in the presence of two or more pathogen strains or species. Moreover, we obtained analytical solutions that allowed us to investigate the disease reduction over the whole range of parameters that includes both qualitative and quantitative host resistance (see Appendix A.5). 


\section{Materials and methods}

We first consider a general case of a mixture with $n$ hosts that is exposed to $n$ pathogens. These could be either different strains (races or pathotypes) of the same pathogen or different pathogen species capable of infecting the same host tissue. The dynamics of the host-pathogen interactions are described by the susceptible-infected model that consists of $2 n$ equations:

$$
\begin{aligned}
\frac{d H_{i}}{d t} & =r_{H}\left(K_{i}-H_{i}\right)-\sum_{k=1}^{n} \beta_{k i} I_{k} H_{i} \\
\frac{d I_{i}}{d t} & =\sum_{k=1}^{n} \beta_{i k} I_{i} H_{k}-\mu I_{i}, i=1, \ldots, n
\end{aligned}
$$

This model is an extension of the model described previously (Mikaberidze et al., 2014) for the case of two pathogen strains infecting a single host variety. This model can be applied to a variety of aerially and splash-dispersed, polycyclic pathogens of cereal crops, such as the fungi and bacteria causing rusts, mildews, blasts, spots and blotches. There are $2 n$ compartments in the model: susceptible hosts $H_{i}$, hosts $I_{i}$ infected by the pathogen $i$, where $i=1, \ldots, n$. The quantities $H_{i}, I_{i}$ represent the total amount of the corresponding host tissue within one field, which could be leaves, stems or grain tissue, depending on the host-pathogen combination.

Susceptible hosts $H_{i}$ grow with the same rate $r_{H}$. Their growth is limited by their "carrying capacities" $K_{i}$, implying limitations in space or nutrients.

The matrix elements $\beta_{i k}$ in Eqs. (1)-(2) constitute the transmission matrix $\mathbf{B}$, an $n \times n$ square matrix [often called WAIFW (Who Acquires Infection From Whom) matrix]. The element $\beta_{i k}$ describes the transmission rate of the pathogen that originates from the infected host of type $i$ and infects the healthy host of variety $k$. We assume that the two host varieties differ only in their susceptibility to the two pathogens, and the two pathogens differ only in their capability to infect 
different hosts, which is reflected in the rate of spore production and the ability of resulting spores to infect additional host tissue. Both host susceptibility and pathogen virulence are described in the model by the transmission rates $\beta_{i k}$. The infected host tissue loses its infectivity (i.e. the ability to produce infectious spores) with the rate $\mu$ ( $\mu^{-1}$ is the average infectious period), which is assumed to be the same for all $I_{i}, i=1, \ldots, n$.

We neglected spatial dependence of pathogen dispersal: every infected host is equally likely to infect every other infected host within the population (often called the "mass-action" approximation). This approximation is valid for air-borne pathogens with long-range dispersal (for example, rusts and mildews), for sufficiently small plot sizes and for a uniform mixture of host varieties. There is evidence that when the overall disease severity is large enough, the disease may develop uniformly across the experimental plots [for example, observations in (Robert et al., 2004) for $Z y$ moseptoria tritici and Puccinia striiformis on wheat]. In other cases this assumption appears to be an idealization (i.e. (Lannou et al., 2008; Mundt, 2009)), especially when looking at the initial stages of an epidemic. In the current study, we are focusing more on the disease severity at the end of the growing season. Understanding of the basic model presented here is a necessary step and a point of reference for further inquiries that will consider autoinfection and spatial dimension explicitly.

We will vary the number of host varieties in the mixture $n$, while keeping the total carrying capacity constant: $K_{\text {tot }}=\sum_{i=1}^{n} K_{i}=n K$. We will consider the total amount of healthy and infected hosts at the infected equilibrium (denoted by an "**"-superscript) of the system of Eqs. (1)(2)

$$
H_{\mathrm{tot}}^{*}=\sum_{i=1}^{n} H_{i}^{*}, I_{\mathrm{tot}}^{*}=\sum_{i=1}^{n} I_{i}^{*} \text {, }
$$

The equilibrium corresponds a fixed point of the system Eqs. (1)-(2) (as explained in the Appendix 
A.1 and A.2) and can achieved over long periods of time, depending on the stability properties of the system. The total disease severity is defined by

$$
y_{\mathrm{tot}}^{*}=\frac{I_{\mathrm{tot}}^{*}}{I_{\mathrm{tot}}^{*}+H_{\mathrm{tot}}^{*}} .
$$

In order to obtain an analytical solution for the disease severity Eq. (4), we consider the transmission matrix of a simple form

$$
\mathbf{B}=\left(\begin{array}{cccc}
\beta_{\mathrm{d}} & \beta_{\mathrm{nd}} & \cdots & \beta_{\mathrm{nd}} \\
\beta_{\mathrm{nd}} & \beta_{\mathrm{d}} & \cdots & \beta_{\mathrm{nd}} \\
\vdots & & \ddots & \\
\beta_{\mathrm{nd}} & \cdots & & \beta_{\mathrm{d}}
\end{array}\right)
$$

Here, every diagonal element of the matrix $\mathbf{B}$ is equal to $\beta_{\mathrm{d}}$ and every non-diagonal element is $\beta_{\text {nd }}$. We generally assume partial specialization, where $\beta_{\mathrm{d}} \geq \beta_{\text {nd }}$. Furthermore, assuming that all healthy and infected hosts start with the same initial conditions, their dynamics will be the same. Hence, the amount of healthy and infected hosts is the same in each compartment $i$ and equal to $H_{p}$ and $I_{p}$, correspondingly. So, we substitute $H_{i}=H_{p}, I_{i}=I_{p}$ in Eqs. (1)-(2) and simplify these equations:

where $\beta_{\text {eff }}=\beta_{\mathrm{d}}+(n-1) \beta_{\mathrm{nd}}$.

$$
\begin{aligned}
\frac{d H_{p}}{d t} & =r_{H}\left(K-H_{p}\right)-\beta_{\mathrm{eff}} I_{p} H_{p}, \\
\frac{d I_{p}}{d t} & =\beta_{\mathrm{eff}} I_{p} H_{p}-\mu I_{p}
\end{aligned}
$$

We also consider the simpler case when two host varieties $H_{1}$ and $H_{2}$ are exposed to two types 
of pathogen: 1 and 2 (we also refer to them as $P_{1}$ and $P_{2}$ ), because mixtures of two host varieties are used most often. The model of susceptible-infected dynamics is described schematically in Fig. 1 and mathematically by the four equations:

$$
\begin{aligned}
\frac{d H_{1}}{d t} & =r_{H}\left(K_{1}-H_{1}\right)-\left(\beta_{11} I_{1}+\beta_{21} I_{2}\right) H_{1}, \\
\frac{d H_{2}}{d t} & =r_{H}\left(K_{2}-H_{2}\right)-\left(\beta_{12} I_{1}+\beta_{22} I_{2}\right) H_{2}, \\
\frac{d I_{1}}{d t} & =\left(\beta_{11} H_{1}+\beta_{12} H_{2}\right) I_{1}-\mu I_{1}, \\
\frac{d I_{2}}{d t} & =\left(\beta_{21} H_{1}+\beta_{22} H_{2}\right) I_{2}-\mu I_{2} .
\end{aligned}
$$

There are four compartments in the model: susceptible hosts $H_{1}$ of variety 1 , susceptible hosts $H_{2}$ of variety 2 , hosts $I_{1}$ infected by pathogen 1 and hosts $I_{2}$ infected by pathogen 2 . One can vary the proportion of host plants of the two varieties by adjusting the ratio of the corresponding seeds to be planted. This is reflected in the change of the ratio $\phi_{1}=K_{1} /\left(K_{1}+K_{2}\right)$ in the model. We assume that the seeds of the two host varieties are well mixed before planting, such that the spatial distribution across the field is uniformly random for both types of plants.

Both host susceptibility and pathogen virulence are described in the model by the four transmission rates $\beta_{11}, \beta_{22}, \beta_{12}$, and $\beta_{21}$. The corresponding transmission matrix has the form

$$
\mathbf{B}=\left|\begin{array}{ll}
\beta_{11} & \beta_{12} \\
\beta_{21} & \beta_{22}
\end{array}\right| .
$$

As before, the first index of matrix elements represents the source of infection and the second index represents the recipient of infection (see Fig. 2). For example, $\beta_{12}$ describes the transmission rate from $I_{1}$ to $H_{2}$. Possible relationships between the elements of the transmission matrix (12) are discussed in Appendix A.5. 
The model describes two distinct limiting cases. First case corresponds to the situation when $I_{1}$ includes the tissue of both hosts infected by pathogen 1 . Similarly, $I_{2}$ includes host tissue of both hosts infected by the pathogen 2 . This formulation assumes that the transmission rate does not depend on the host variety of the source of infection, but only depends on the host variety of the recipient of infection. In other words, under this assumption, the spore production rate and the quality of spores produced depend on the pathogen genotype, but not on the host genotype. But the infection efficiency (or infection success) of a spore depends on the host genotype on which it lands. Second case is realized when $I_{1}$ includes the tissue of host 1 that is infected by any one of the two pathogens. Similarly, $I_{2}$ includes the tissue of host 2 that is infected by any one of the two pathogens. This represents the other limiting case, when the spore production rate and the quality of spores produced depend on the host genotype, but not on the pathogen genotype. We In order to relax these assumptions, one needs to subdivide each of $I_{1}$ and $I_{2}$ into two compartments, according to the type of host tissue infected (first case), or according to the infecting pathogen (second case).

In this simplified case, the equilibrium disease severity Eq. (4) has the form

$$
y_{\mathrm{tot}}^{*}=\left(I_{1}^{*}+I_{2}^{*}\right) /\left(I_{1}^{*}+I_{2}^{*}+H_{1}^{*}+H_{2}^{*}\right) .
$$

We use $y_{\mathrm{tot}}^{*}$ that corresponds to the disease severity close to the end of the growing season, to quantify the efficacy of host mixtures in terms of disease reduction. Previous modeling studies (Gumpert et al., 1987; Gumpert, 1989; Gumpert and Geiger, 1995) considered the other limiting case by assuming that the amount of disease is growing exponentially over time. 


\section{Results}

First, we present the outcomes of the general model that describes many pathogen strains and host varieties and determine an optimal number of components in a host mixture. Next, we determine proportions of hosts in the mixture that will minimize the disease.

\section{What is the optimal number of components to use in a host mixture?}

In order to answer this question, we consider a mixture of $n$ hosts exposed to $n$ pathogens. and use the mathematical framework of Eqs. (6)-(7). In the case of partial specialization all elements of the transmission matrix $\mathbf{B}$ are positive. All the diagonal elements are equal to $\beta_{\mathrm{d}}$ and the nondiagonal ones are equal to $\beta_{\text {nd }}$, with $\beta_{\mathrm{d}}>\beta_{\text {nd }}>0$ (see Eq. (5)). In this case, we determined (see Appendix A.3) the analytical expression for the total disease severity at the infected equilibrium, assume that every host variety is planted at the same proportion, i. e. $K_{i}=K$ :

$$
y_{\text {tot }}^{*}(n)=r_{H} \frac{\left(\beta_{\mathrm{d}}+(n-1) \beta_{\mathrm{nd}}\right) K_{\mathrm{tot}}-\mu n}{n \mu(\mu-r)+r K_{\mathrm{tot}}\left(\beta_{\mathrm{d}}+(n-1) \beta_{\mathrm{nd}}\right)} \text {. }
$$

Using this expression, we plotted in Fig. 3 the disease severity as a function of the number of components in the mixture $n$. Panel (a) illustrates the case of a pathogen with the high rate of transmission and panel (b) shows the case a pathogen with the intermediate rate of transmission. The grey solid curves represent the homogeneous case when $\beta_{\mathrm{nd}}=\beta_{\mathrm{d}}>0$, i.e. no specialization, every pathogen strain or species is equally likely to infect every host. Evidently, in this case the disease severity is independent of the number of mixture components. In all other cases considered in Fig. 3, the disease severity decreases with $n$. The black solid curves in Fig. 3 illustrate the case of full specialization, when $\beta_{\mathrm{nd}}=0, \beta_{\mathrm{d}}>0$. In this case, the disease severity decreases steeply 
with increasing $n$, eventually reaching zero. The dashed curves in Fig. 3 correspond to intermediate cases with different degrees of partial specialization. As the degree of host specialization increases, the decrease in disease severity becomes stronger.

Can one eradicate the disease by adding a large enough number of components to the host mixture? As we increase the number of components in the host mixture, each pathogen strain can infect less of its preferred host. At the limit of very large $n$, the amount of preferred host tissue available for each pathogen strain is so small that they are not able to survive only on it. Therefore, whether we can eradicate the disease depends on the ability of pathogen strains to survive on hosts that are not their favorite. This is determined by the parameter $R_{0 \text { nd }}=\beta_{\text {nd }} K_{\text {tot }} / \mu$, which is the basic reproductive number of pathogen strains as a whole in the absence of their preferred hosts. If $R_{0 \text { nd }}>1$, then pathogen strains can survive in the absence of their preferred hosts. In this case, disease severity tends to a constant positive value at large $n$ and never decreases to zero (dashdotted curve in Fig. 3). In contrast, when $R_{0 \text { nd }}<1$, pathogen strains die out in the absence of their preferred hosts.

We take the the limit of very large $n$ in Eq. (14) and find that the disease severity is proportional to $R_{0 \text { nd }}-1$ in this case:

$$
y_{\mathrm{tot}}^{*}(n)_{n \rightarrow \infty}=r_{H} \frac{R_{0 \mathrm{nd}}-1}{\mu+r_{H}\left(R_{0 \mathrm{nd}}-1\right)},
$$

where $R_{0 \text { nd }}=\beta_{\text {nd }} K_{\text {tot }} / \mu$ is the basic reproductive number of pathogen strains overall in the absence of their preferred hosts. It follows from Eq. (15) that if $R_{0 \text { nd }} \leq 1$, then the disease severity will eventually reach (or approach) zero as we increase $n$. However when $R_{0 \text { nd }}>1$, the disease severity will approach a constant positive value given by Eq. (15). This means that, by increasing the number of components in the mixture, we decrease (eventually to zero) the impact of hostspecialized infections characterized by rate $\beta_{\mathrm{d}}$. However, the impact of non-specialized infections 
characterized by $\beta_{\text {nd }}$ remains unchanged with the corresponding severity given by Eq. (15).

From the expression for the disease severity in Eq. (14), one can determine the optimal number of components to use in the mixture. One way to do this is to define an economically acceptable disease severity, $y_{\mathrm{acc}}$, (for example $5 \%$ ), and then determine the number of components in the mixture that decrease the disease severity down to $y_{\text {acc. Th }}$ This is done by solving Eq. (14) with respect to $n$. As a result, we obtain

$$
n_{\mathrm{opt} 1}=r_{H} K_{\mathrm{tot}} \frac{\left(\beta_{d}-\beta_{\mathrm{nd}}\right)\left(1-y_{\mathrm{acc}}\right)}{\mu\left(r_{H}+y_{\mathrm{acc}}\left(\mu-r_{H}\right)\right)-r_{H} \beta_{\mathrm{nd}} K_{\mathrm{tot}}\left(1-y_{\mathrm{acc}}\right)} .
$$

Here, $n_{\mathrm{opt} 1}$ is the number of mixture components at which the disease severity $y_{\mathrm{acc}}$ is reached. This is illustrated in Fig. 3, where the horizontal dashed line corresponds to $y_{\text {acc }}=5 \%$. The values of $n$ at which this line intersects with disease severity curves correspond to optimum $n_{\text {opt1 }}$ given by Eq. (16). The optimum shifts to larger values with decreasing degrees of specialization [e.g. from $n_{\mathrm{opt} 1}=9$ for the solid curve corresponding to full specialization to $n_{\mathrm{opt} 1}=16$ for the dashed curve representing partial specialization in Fig. 3(a)]. Also, the optimum number of components is proportional to the total host population size $K_{\text {tot }}$.

Another way to determine an optimal number of mixture components uses the fact that $y^{*}(n)$ decreases with $n$, but also considers that the rate of this decrease (i. e. the derivative $\frac{d y^{*}(n)}{d n}$ ) decreases with $n$. Hence, the benefit of adding one more component to a mixture that already has $n$ components decreases with increasing $n$. Because of this, the dependence $y^{*}(n)$ eventually saturates to a constant value given by Eq. (15). Therefore, one can define a minimum decrease in disease severity due to adding one more host variety to the mixture $\Delta y_{\min }$ that is still economically plausible. The number of mixture components at this minimum is optimal, i. e. $n=n_{\mathrm{opt} 2}$. Mathematically, $n_{\mathrm{opt} 2}$ can be found from the equation $y_{\mathrm{tot}}^{*}\left(n_{\mathrm{opt} 2}-1\right)-y_{\mathrm{tot}}^{*}\left(n_{\mathrm{opt} 2}\right)=\Delta y_{\mathrm{min}}$, where $y_{\mathrm{tot}}^{*}(n)$ is given by 
Eq. (14). The solution reads as

$$
n_{\mathrm{opt} 2}=\frac{\sqrt{\Delta y}\left[\mu^{2}-r_{H}\left(K_{\mathrm{tot}}\left(2 \beta_{\mathrm{d}}-3 \beta_{\mathrm{nd}}\right)+\mu\right)\right]+\sqrt{4\left(\beta_{\mathrm{d}}-b_{\mathrm{nd}}\right) r_{H} K_{\mathrm{tot}} \mu^{2}+\Delta y C}}{2 \sqrt{\Delta S} C^{2}},
$$

where $C=\mu^{2}+r_{H}\left(\beta_{\text {nd }} K_{\text {tot }}-\mu\right)$. This is also illustrated in Fig. 3, where the dotted vertical lines shows $n_{\mathrm{opt} 2}=3$ [panel (a)] and $n_{\mathrm{opt} 2}=2$ [panel (b)] that correspond to the severity curves for the case of strong partial specialization (dashed curves). When the degree of specialization is increased further up to full specialization (solid curve), $n_{\mathrm{opt} 2}$ shifts to the larger value of four.

We expect mixtures to be more effective against pathogens with intermediate and low transmission [cf. panels (a) and (b) in Fig. 3]. In Fig. 3(b) a mixture with three components not only decreased the disease below the acceptable level [optimum number of components, according to Eq. (16)], but even eradicated the pathogen. A two-component mixture provided an economical optimum, according Eq. (17). In contrast, for pathogens with high transmission [Fig. 3(a)], mixtures with more components need to be used to reach the optimal effects.

The optimum number of components in the mixture, defined according to Eq. (16), can only be found if the acceptable severity $y_{\text {acc }}$ can be reached by increasing $n$ (that is when $R_{0 \text { nd }}<1$ ). This restriction is removed in the definition based on Eq. (17). But even in cases when $y_{\text {acc }}$ can be reached by increasing $n$, the second definition seems to be more plausible, since it incorporates the economic costs of introducing an additional component into the mixture. However, it does not ensure that the disease will be reduced down to an acceptable value. Hence, additional disease control measures (e.g. applications of fungicides) may need to be implemented in order to further reduce the disease. 


\section{Is there an optimal mixture of host varieties?}

Planting a mixture of host varieties provides an additional parameter that can be adjusted, namely the proportions of the varieties in the mixture. Does planting a mixture of hosts reduce the total amount of disease compared to the case of monoculture stands? Furthermore, is there an optimal proportion of the host varieties at which the amount of disease is minimized? Answers to these questions depend on the relationships between the elements of the transmission matrix $\mathbf{B}$.

We calculate the disease severity at equilibrium $y^{*}$ [Eq. (13)] as a function of the proportion of the host variety 1 in the mixture $\phi_{1}=K_{1} / K$ [see Fig. 4(a)]. The quantity $\phi_{1}$ is varied from zero to one, while keeping the total carrying capacity of hosts $K=K_{1}+K_{2}$ constant.

When each pathogen can infect both hosts equally well (i.e. $\beta_{12}=\beta_{11}, \beta_{21}=\beta_{22}$, no specialization), disease severity does not depend on $\phi_{1}$ [horizontal dashed curve in Fig. 4(a)]. The same outcome is observed when the host-pathogen interaction follows the pure gene-for-gene scheme (scenario (A) in Appendix A.5), i.e. $\beta_{11}=\beta_{21}=\beta_{22}>0, \beta_{12}=0$ [horizontal dashed curve in Fig. 4(a)]. We used the values of the transmission rates, which satisfy $\beta_{22}>\beta_{11}$. Hence, pathogen 2 is fitter than pathogen 1 and dominates the population and at any value of $\phi_{1}$ [the two horizontal dashed curves overlap completely in Fig. 4(b)].

In the case of a single pathogen infecting a mixture of hosts with different degrees of susceptibility $\left(\beta_{22}=\beta_{12}>\beta_{11}=\beta_{21}\right)$, the disease severity decreases linearly with $\phi_{1}$. In this case, simply using a monoculture with the more disease-resistant host variety $\left(\phi_{1}=1\right)$ would reduce the disease most strongly [green dashed-dotted curve in Fig. 4(a)]. This is in agreement with findings of an experiment, in which a mixture of a susceptible and resistant barley variety was infected by barley powdery mildew (caused by Blumeria graminis f. sp. hordei) reported in (Finckh et al., 2000). In this study the disease reduction was found to decrease linearly with the proportion of the 
susceptible variety in the mixture.

The picture changes if there is a degree of specialization of pathogen strains or species to host varieties $\left(\beta_{22}, \beta_{11}>\beta_{12}, \beta_{21}\right.$, scenario (D) in Appendix A.5). In this case the disease severity $y^{*}$ first decreases with $\phi_{1}$, then reaches a constant value, and after that increases again. Thus, the disease is reduced over a range of intermediate values of $\phi_{1}$ (solid and dotted curves in Fig. 4(a)). The magnitude of this reduction increases with the degree of specialization and reaches a maximal value at full specialization (solid red curve). Also, the range of $\phi_{1}$-values, over which the proportion of disease remains minimal, increases with the degree of specialization [cf. solid and dotted curves in Fig. 4(a)].

The ranges over which the frequency of pathogen 2 remains constant or changes as a function of the cropping ratio $\phi_{1}$ correspond to the ranges of stability of different fixed points of the model system Eqs. (8)-(11). This can be seen from Fig. 4(b), where the frequency $f_{2}$ of pathogen 2 is shown versus $\phi_{1}$. In the region where $y^{*}$ decreases with $\phi_{1}$, pathogen 2 dominates the population $\left(f_{2}=1\right)$. In the region where $y^{*}$ stays constant, the two pathogens co-exist, but the frequency of pathogen 2 decreases with $\phi_{1}$ until it reaches zero. This occurs at the border, where another fixed point becomes stable, the one corresponding to pathogen 1 dominating the population $\left(f_{2}=1\right)$. Here, the disease severity increases with $\phi_{1}$.

Why does the disease severity decrease with $\phi_{1}$ at small values of $\phi_{1}$ ? In this parameter range, pathogen 2 dominates the population in the long term. Since pathogen 2 specializes on host 2, it develops best when only host 2 is planted, i. e. at $\phi_{1}=0$. By adding a small amount of host 1 to the mixture, we create suboptimal conditions for pathogen 2: it is still able to outcompete pathogen 1 , but since there is less of its preferred host tissue, the resulting disease severity is smaller. A similar explanation holds for the increase of disease severity with $\phi_{1}$ at large values of $\phi_{1}$.

Why does the disease severity stay constant over a range of intermediate values of $\phi_{1}$ ? This 
range corresponds to co-existence of the two pathogens. Since there is a degree of specialization, by increasing $\phi_{1}$ we make pathogen 1 more fit while pathogen 2 becomes less fit. These two changes compensate each other, so that the total disease severity, which includes both pathogen strains, remains the same.

Thus, mixing host varieties reduces the overall disease severity if each of the pathogens performs better on its preferred host. In this case, an optimal proportion of host varieties in the mixture lies in the intermediate range, over which the two pathogens exhibit stable co-existence. This result is in agreement with previous theoretical studies (Lively, 2010) and also explains some experimental findings (Zhan and McDonald, 2013).

We also investigated the time dependence of the disease severity before the equilibrium is reached (see Appendix 4, Figure A.1) by numerically solving the system of Eqs. (8)-(11). The solutions indicate that the optimal suppression of disease at intermediate cropping ratios, $\phi_{1}$, appears much before the equilibrium is approached. Also, the optimal range of $\phi_{1}$-values at the equilibrium that we determined analytically is indicative of the optimal range in the early phases of the dynamics.

Further, our results indicate that the benefit of mixing two host varieties increases with decreasing the pathogen's basic reproductive number. To illustrate this effect, we quantified this benefit using the ratio between the mean disease severity in pure stands and the disease severity of the $50 / 50$ host mixture. We considered this quantity as a function of the mean basic reproductive number of the two pathogens. This reveals, that mixing host varieties can be an effective measure to control pathogens (with the reduction of disease severity by more than $20 \%$ ) with intermediate values of $R_{0}$ (between 5 and 20, for example, Zymoseptoria tritici has $R_{0}$ of about 10), but will bring only about $10 \%$ reduction in disease severity when contolling diseases with high $R_{0}$ 's, such as stripe rust of wheat (where $R_{0}$ is about 50 (Segarra et al., 2001)). 
In addition, within the range of maximal overall suppression of disease, the ratio of the two pathogens can be controlled by varying the proportion of hosts in the mixture [Fig. 4(a) and (b)]. This can be useful, if one of the pathogens is much less desirable, for example, because of mycotoxin production or the risk of fungicide resistance.

\section{Discussion}

We have shown that when a population of crop plants is exposed to two host-specialized pathogen strains or species, the overall severity of both diseases is smaller in the mixture of two host varieties than in either of the pure stands. We obtained analytical expressions for the disease reduction which allowed us to quantify it across the whole range of parameters. These findings may help to identify crop cultivars to be deployed in mixtures that will successfully control diseases prevalent in a given region. The overall disease severity can be minimized over a range of mixing ratios. The two pathogens coexist in this range and further adjusting the mixing ratio within this range makes it possible to control the relative abundance of each pathogen. This can be useful when one of the pathogens is less desirable, for example due to mycotoxin production or fungicide resistance, while a certain amount of the other pathogen can be tolerated. Alternatively, the mixing ratio can be adjusted within this optimal range to increase the economic output of the crop, if the two host varieties differ in their quality or commercial value.

We also generalized the model to describe host mixtures with more than two components. We find that when there is a degree of host specialization, the overall disease severity decreases with the number of components in the mixture. The more specialized the host-pathogen pairs are, the stronger is the decrease in the disease severity. Based on this understanding, we proposed ways to determine economically optimal numbers of components in host mixtures. Furthermore, this 
more general framework is capable of describing many hosts exposed to many pathogen strains or species and can also be used to better understand plant-pathogen dynamics in natural ecosystems, such as Linum marginale-Melampsora lini (Thrall et al., 2002), or Plantago lanceolataPodosphaera plantaginis (Laine, 2007). Local adaptation was observed in these natural interactions (Thrall et al., 2002; Laine, 2007) and also modelled within a simplified metapopulation framework (Papaïx et al., 2014). Hence, the insight we gained in the case of partial specialization may advance our understanding of evolutionary forces operating in these wild plant-pathogen systems.

It is desirable to study the benefit of mixing host varieties representing the whole range of values of the matrix elements of $\mathbf{B}$ (see Appendix A.5 for the discussion of plausible relationships between the matrix elements). We have done this here by obtaining analytical expressions for the disease severity and frequencies of pathogens as functions of the matrix elements $\beta_{i j}$ and other model parameters (see Appendix A.3). This is an advantage of our study with compared to previous theoretical investigations that assumed a "pure GFG" interaction, without fitness costs associated with losing effectors (Ohtsuki and Sasaki, 2006; van den Bosch and Gilligan, 2003; Lo Iacono et al., 2013), or that assumed full specialization (Lively, 2010), where each pathogen can only infect its preferred host and is unable to infect any other hosts (also called the "matching alleles" model (King and Lively, 2012)). The latter scenario seems to represent only a hypothetical limiting case, because it requires full resistance, which is unlikely given the simultaneous presence of many pairs of R- and E-proteins. In contrast, partial specialization (scenario (D)), when the diagonal elements $\beta_{11}$ and $\beta_{22}$ are larger than non-diagonal ones $\beta_{12}$ and $\beta_{21}$, but the non-diagonal ones are still significantly larger than zero, seems to be the most generic case. This is because it arises from a ubiquitous GFG-type of interaction with many R-proteins present in the host, many corresponding E-proteins present in the pathogen, as well as fitness costs for the pathogen due to 
elimination or modification of E-proteins and fitness costs for the host due to having unnecessary R-proteins.

Some modeling studies considered the effect of varying the proportion of mixture components and partial specialization in host mixtures (Gumpert and Geiger, 1995), but they did not investigate the dependence of the mixture efficacy on the degree of specialization. To the best of our knowledge, the optimal number of components in a host mixture and the optimal ratios of two-component mixtures as functions of the pathogen's reproductive ability and the degree of host specialization have not yet been quantified in the existing modeling literature. Here, we obtained analytical expressions for these quantities that allow to investigate the mixture efficacy in the whole range of parameters and also understand the underlying mechanisms of disease reduction in mixtures.

Mixtures and pure stands of several wheat cultivars were inoculated using a mixture of two wheat stripe rust races in a series of field experiments (Finckh and Mundt, 1992a,b). The pathogen population exhibited host specialization with respect to two of the host mixtures. The pattern of disease severity corresponding to different proportions of host cultivars in mixtures corresponds qualitatively to our model predictions (i. e. solid curve in Fig. 4(a) in the case of host specialization and dash-dotted curve for mixtures of susceptible and resistant cultivars). Our model also predicts coexistence of host-specialized pathogen races in the intermediate range of mixing ratios. This could be tested in experiments similar to (Finckh and Mundt, 1992a,b) by measuring the frequencies of the different pathogen races in the experimental plots. However, interactions between plant genotypes had considerable effect on the disease severity in host mixtures (Finckh and Mundt, $1992 a, b)$ and need to be included in the model in order to achieve quantitative agreement.

Four distinct mechanisms of disease reduction by host mixtures are described in the literature (Chin and Wolfe, 1984; Wolfe, 1985; Finckh et al., 2000): (i) the effect of reduced density of susceptibles; (ii) the "barrier effect"; (iii) induced resistance (Goleniewski, 1996); and (iv) com- 
petition between pathogens. In scenario (i) the disease is reduced in the mixture simply because it has less of the susceptible variety than the susceptible pure stand. This "reduced density" effect can be observed most clearly by comparing the amount of disease in two pure stands of the susceptible variety, which differ only in planting density (Chin and Wolfe, 1984). The introduction of the resistant variety further reduces the disease in the mixture (scenario (ii)), because the transmission between susceptible hosts is hindered (a resistant "barrier" is created between adjacent susceptible plants). Induced resistance (scenario (iii)) takes place when spores of an avirulent pathogen activate a host resistance mechanism that is also effective against another pathogen (or another race of the same pathogen), which is normally able to infect the host (Chin and Wolfe, 1984; Lannou et al., 1995, 2005). Finally, in scenario (iv) mixing host cultivars is expected to make the pathogens compete with each other for host tissue (Finckh et al., 2000; Ohtsuki and Sasaki, 2006).

The "reduced density" effect originally referred to the mixture of a susceptible and a resistant variety (Chin and Wolfe, 1984). Hence, it cannot lead to a disease level lower than in the pure stand of the resistant variety. Here we extended the notion of the "reduced density" effect to the case of two or more host-specialized pathogen strains or species. For example, this may correspond to host 1 being susceptible to pathogen 1, but resistant to pathogen 2 and host 2 being susceptible to pathogen 2, but resistant to pathogen 1 . We find that it is only in such cases that disease level in the mixture is lower than in both pure stands.

Our model does not include the "barrier" effect, since it does not explicitly consider the spatial dependence of pathogen dispersal (see Sec.). Also, induced resistance (Chin and Wolfe, 1984; Lannou et al., 1995, 2005) was not considered. Therefore, we likely underestimate the effect of host mixtures on disease reduction. Moreover, interesting effects of adjusting other landscape variables than the cropping ratio $\phi_{1}$, such as the host patch size and the size of initial disease foci, were observed in recent field experiments on wheat stripe rust (Mundt et al., 2011; Estep 
et al., 2014). These developments stimulate the extension of the basic model for host mixtures presented here using a spatially-explicit approach. In this way, a unified mathematical framework for description of the effect of host mixtures on plant disease can be developed on the basis of the model presented here. This would allow one to better understand the relative contributions of each of these effects in disease reduction and design better host mixtures.

\section{Acknowledgements}

AM and SB gratefully acknowledge support by the ERC advanced grant PBDR 268540. AM would like to thank Gabriel Leventhal for helpful discussions.

\section{References}

Browning, J., and K. Frey. 1969. Multiline cultivars as a means of disease control. Annual Review of Phytopathology 14:355-82.

Brunner, P., F. Stefanato, and B. McDonald. 2008. Evolution of the CYP51 gene in Mycosphaerella graminicola : evidence for intragenic recombination and selective replacement. Molecular plant pathology 9:305-316.

Chin, K., and M. Wolfe. 1984. The spread oi Erysiphe graminis f . sp . hordei in mixtures of barley varieties. Plant Pathology 33:89-100.

Cowger, C., and C. C. Mundt. 2002. Effects of Wheat Cultivar Mixtures on Epidemic Progression of Septoria Tritici Blotch and Pathogenicity of Mycosphaerella graminicola. Phytopathology 92:617-23. 
Estep, L. K., K. E. Sackett, and C. C. Mundt. 2014. Influential disease foci in epidemics and underlying mechanisms: A field experiment and simulations. Ecological Applications in press:dx.doi.org/10.1890/13-1408.1.

Finckh, M., E. Gacek, H. Goyeau, C. Lannou, U. Merz, C. C. Mundt, L. Munk, J. Nadziak, A. C. Newton, C. de Vallavieille-Pope, and M. S. Wolfe. 2000. Cereal variety and species mixtures in practice, with emphasis on disease resistance. Agronomie 20:813-837.

Finckh, M., and C. Mundt. 1992a. Plant competition and disease in genetically diverse wheat populations. Oecologia 91:82-92.

Finckh, M., and C. Mundt. 1992b. Stripe rust, yield, and plant competition in wheat cultivar mixtures. Phytopathology 82:905.

Garrett, K. A., and C. C. Mundt. 1999. Epidemiology in mixed host populations. Phytopathology 89:984-90.

Goleniewski, G. 1996. Modelling Cultivar Mixtures Using SEIR Compartmental Models. Biometrical Journal 38:281-297.

Gumpert, F. M. 1989. Measuring disease progress in pure and mixed stands of plant cultivars. Phytopathology 79:968.

Gumpert, F. M., and H. H. Geiger. 1995. Long-term strategies of disease reduction by suse of cultivar mixtures I. Exponential growth of epidemics. Journal of Plant Diseases and Protection 102:191-202.

Gumpert, F. M., H. H. Geiger, and S. U. 1987. A mathematical model of the epidemics in 
homogeneous and heterogeneous host stands. Journal of Plant Diseases and Protection 94:206215.

Huang, C., Z. Sun, H. Wang, Y. Luo, and Z. Ma. 2012. Effects of wheat cultivar mixtures on stripe rust: A meta-analysis on field trials. Crop Protection 33:52-58.

King, K. C., and C. M. Lively. 2012. Does genetic diversity limit disease spread in natural host populations? Heredity 109:199-203.

Kou, Y., and S. Wang. 2010. Broad-spectrum and durability: understanding of quantitative disease resistance. Current opinion in plant biology 13:181-5.

Laine, A.-L. 2007. Detecting local adaptation in a natural plant-pathogen metapopulation: a laboratory vs. field transplant approach. Journal of evolutionary biology 20:1665-73.

Lannou, C., P. Hubert, and C. Gimeno. 2005. Competition and interactions among stripe rust pathotypes in wheat-cultivar mixtures. Plant Pathology 54:699-712.

Lannou, C., S. Soubeyrand, L. Frezal, and J. Chadoeuf. 2008. Autoinfection in wheat leaf rust epidemics. The New phytologist 177:1001-11.

Lannou, C., C. Vallavieille-Pope, and H. Goyeau. 1995. Induced resistance in host mixtures and its effect on disease control in computer-simulated epidemics. Plant Pathology 44:478-489.

Lehman, J. S., and G. Shaner. 1997. Selection of Populations of Puccinia recondita f. sp. tritici for Shortened Latent Period on a Partially Resistant Wheat Cultivar. Phytopathology 87:170-6.

Lively, C. M. 2010. The Effect of Host Genetic Diversity on Disease Spread. The American naturalist 175:E149-52. 
Lo Iacono, G., F. van den Bosch, and C. a. Gilligan. 2013. Durable Resistance to Crop Pathogens: An Epidemiological Framework to Predict Risk under Uncertainty. PLoS computational biology 9:e1002870.

McDonald, B. A. 2014. Using dynamic diversity to achieve durable disease resistance in agricultural ecosystems. Tropical Plant Pathology 39:191-196.

McDonald, B. A., and C. Linde. 2002. Pathogen population genetics, evolutionary potential, and durable resistance. Annual Review of Phytopathology 40:349-79.

Mikaberidze, A., B. A. McDonald, and S. Bonhoeffer. 2014. Can high risk fungicides be used in mixtures without selecting for fungicide resistance? Phytopathology 104:324-331.

Mundt, C., C. Cowger, and K. Garrett. 2002. Relevance of integrated disease management to resistance durability. Euphytica 124:245-252.

Mundt, C., P. Hayes, and C. Schön. 1994. Influence of barley variety mixtures on severity of scald and net blotch and on yield. Plant pathology 43:356-361.

Mundt, C., K. Sackett, and L. Wallace. 2011. Landscape heterogeneity and disease spread: experimental approaches with a plant pathogen. Ecological Applications 21:321-328.

Mundt, C. C. 2002. Use of multiline cultivars and cultivar mixtures for disease management. Annual review of phytopathology 40:381-410.

Mundt, C. C. 2009. Importance of autoinfection to the epidemiology of polycyclic foliar disease. Phytopathology 99:1116-20.

Newton, A., G. Begg, and J. Swanston. 2009. Deployment of diversity for enhanced crop function. Annals of Applied Biology 154:309-322. 
Newton, A., R. Ellis, C. Hackett, and D. Guy. 1997. The effect of component number on Rhynchosporium secalis infection and yield in mixtures of winter barley cultivars. Plant Pathology 45:930-938.

Newton, A., and D. Guy. 2011. Scale and spatial structure effects on the outcome of barley cultivar mixture trials for disease control. Field Crops Research 123:74-79.

Ning, L. I., J. I. A. Shao-feng, X.-n. Wang, X.-y. Duan, Y.-1. Zhou, Z.-h. Wang, and G.-d. Lu. 2012. The Effect of Wheat Mixtures on the Powdery Mildew Disease and Some Yield Components. Journal of Integrative Agriculture 11:611-620.

Ohtsuki, A., and A. Sasaki. 2006. Epidemiology and disease-control under gene-for-gene plantpathogen interaction. Journal of theoretical biology 238:780-94.

Papaïx, J., J. J. Burdon, C. Lannou, and P. H. Thrall. 2014. Evolution of pathogen specialisation in a host metapopulation: joint effects of host and pathogen dispersal. PLoS computational biology 10:e1003633.

Papaïx, J., H. Goyeau, P. Du Cheyron, H. Monod, and C. Lannou. 2011. Influence of cultivated landscape composition on variety resistance: an assessment based on wheat leaf rust epidemics. The New phytologist 191:1095-107.

Parlevliet, J. 2002. Durability of resistance against fungal, bacterial and viral pathogens; present situation. Euphytica 124:147-156.

Poland, J. a., P. J. Balint-Kurti, R. J. Wisser, R. C. Pratt, and R. J. Nelson. 2009. Shades of gray: the world of quantitative disease resistance. Trends in plant science 14:21-9.

Robert, C., M.-O. Bancal, P. Nicolas, C. Lannou, and B. Ney. 2004. Analysis and modelling of 
effects of leaf rust and Septoria tritici blotch on wheat growth. Journal of experimental botany 55:1079-94.

Segarra, J., M. J. Jeger, and F. van den Bosch. 2001. Epidemic dynamics and patterns of plant diseases. Phytopathology 91:1001-10.

Singh, R. P., D. P. Hodson, J. Huerta-Espino, Y. Jin, S. Bhavani, P. Njau, S. Herrera-Foessel, P. K. Singh, S. Singh, and V. Govindan. 2011. The emergence of Ug99 races of the stem rust fungus is a threat to world wheat production. Annual review of phytopathology 49:465-81.

Smithson, J., and J. Lenne. 1996. Varietal mixtures: a viable strategy for sustainable productivity in subsistence agriculture. Annals of Applied Biology 128:127-158.

Stuthman, D., K. Leonard, and J. MillerGarvin. 2007. Breeding Crops for Durable Resistance to Disease. Advances in Agronomy 95:319-367.

Thrall, P. H., J. J. Burdon, and J. D. Bever. 2002. Local adaptation in the Linum marginaleMelampsora lini host-pathogen interaction. Evolution; international journal of organic evolution 56:1340-51.

Torriani, S. F., P. C. Brunner, B. A. McDonald, and H. Sierotzki. 2009. QoI resistance emerged independently at least 4 times in European populations of Mycosphaerella graminicola. Pest Manag. Sci. 65:155-62.

van den Bosch, F., and C. A. Gilligan. 2003. Measures of durability of resistance. Phytopathology 93:616-25.

Walters, D. R., A. Avrova, I. J. Bingham, F. J. Burnett, J. Fountaine, N. D. Havis, S. P. Hoad, G. Hughes, M. Looseley, S. J. P. Oxley, A. Renwick, C. F. E. Topp, and A. C. Newton. 2012. 
Control of foliar diseases in barley: towards an integrated approach. European Journal of Plant Pathology 133:33-73.

Wolfe, M. 1985. The current status and prospects of multiline cultivars and variety mixtures for disease resistance. Annual Review of Phytopathology 23:251-73.

Wolfe, M. S. 2000. Crop strength through diversity. Nature 20:681-2.

Zhan, J., and B. a. McDonald. 2013. Experimental measures of pathogen competition and relative fitness. Annual review of phytopathology 51:131-53.

Zhu, Y., H. Chen, J. Fan, Y. Wang, Y. Li, J. Chen, S. Yang, L. Hu, H. Leung, T. W. Mew, P. S. Teng, Z. Wang, and C. C. Mundt. 2000. Genetic diversity and disease control in rice. Nature 406:718-22.

\section{Figure captions}

Figure 1. Sheme of the model equations (8)-(11).

Figure 2. Scheme of the host-pathogen interaction. "+" refers to full susceptibility, "-" refers to full resistance to disease, and these signs correspond to a "pure" gene-for-gene (GFG) interaction. The transmission matrix $\beta_{i j}, i, j=1,2$ represents a more general description with "pure" GFG $\left(\beta_{11}=\beta_{22}=\beta_{21}>0 ; \beta_{12}=0\right)$ and full host specialization $\left(\beta_{11}, \beta_{22}>0 ; \beta_{12}=\beta_{21}=0\right)$ as limiting cases.

Figure 3. Disease severity at the infected equilibrium versus the number of components in the host mixture plotted according to Eq. (14) in the case of no specialization (grey solid), full 
specialization (solid), partial specialization with the specialization index $\sigma=\beta_{\text {nd }} / \beta_{\mathrm{d}}=0.5$ (dashdotted) and $\sigma=0.05$ (dashed). Paramer values: (a) pathogen with high transmission $\beta_{\mathrm{d}}=2$; (b) pathogen with low transmission $\beta_{\mathrm{d}}=0.5$. The rest of parameters are the same in (a) and (b): $K_{\text {tot }}=1, \mu=0.2, r=0.1$. Dotted horizontal curve shows an example of a maximum disease severity, $S_{\text {acc }}=5 \%$, that is still economically acceptable. Dotted vertical lines show the optimal number of components $n_{\mathrm{opt} 2}=3$ [panel (a)] and $n_{\mathrm{opt} 2}=2$ [panel (b)], according to Eq. (17) taking $\Delta S=10 \%$, for the dashed curves.

Figure 4. Disease severity $y^{*}$ (upper panel) and the frequency $f_{2}^{*}=I_{2}^{*} /\left(I_{1}^{*}+I_{2}^{*}\right)$ of pathogen 2 (lower panel) at equilibrium as functions of the proportion of host 1 in the mixture $\phi_{1}=K_{1} /\left(K_{1}+\right.$ $K_{2}$ ), according to Eqs. (A.16), (A.17). Parameter values: $\beta_{11}=6$ (unless specified otherwise below), $\beta_{22}=8, K=K_{1}+K_{2}=1, r=0.2, \mu=1$. The non-diagonal elements of the infection matrix $\mathbf{B}$ determine the degree of specialization: (a) full specialization $\beta_{12}=\beta_{21}=0$ (red dotted); (b) small degree of specialization $\beta_{12}=\beta_{21}=0.9$ (blue, solid); (c) no specialization $\beta_{12}=\beta_{11}=$ $6, \beta_{21}=\beta_{22}=8$ (black, upper); (d) "pure" gene-for-gene interaction $\beta_{11}=\beta_{21}=\beta_{22}=8$ $\beta_{12}=0$ (yellow, upper); (e) single pathogen $\beta_{11}=\beta_{21}=6, \beta_{22}=\beta_{12}=8$ (green, dash-dotted). Cases (c) and (d) correspond to the upper horizontal lines and overlap completely. 


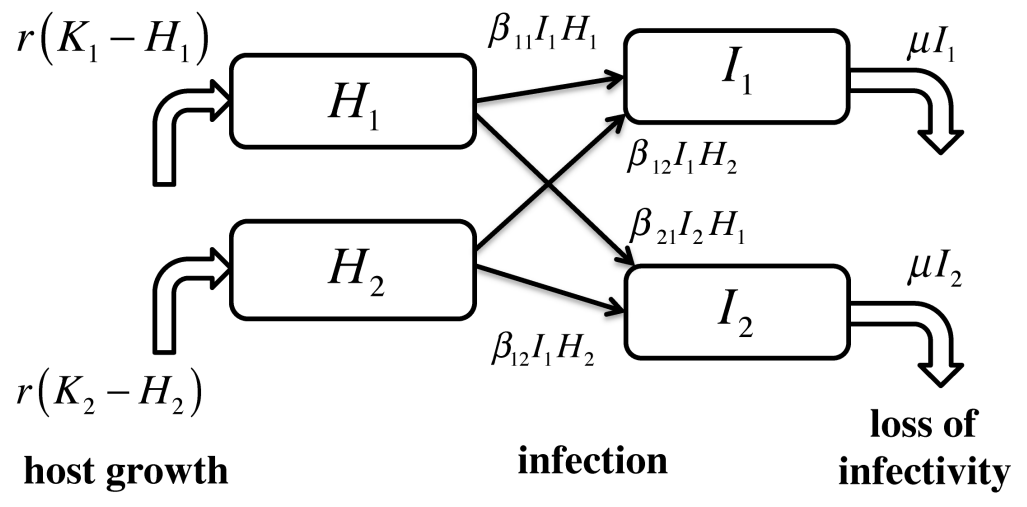

Figure 1 


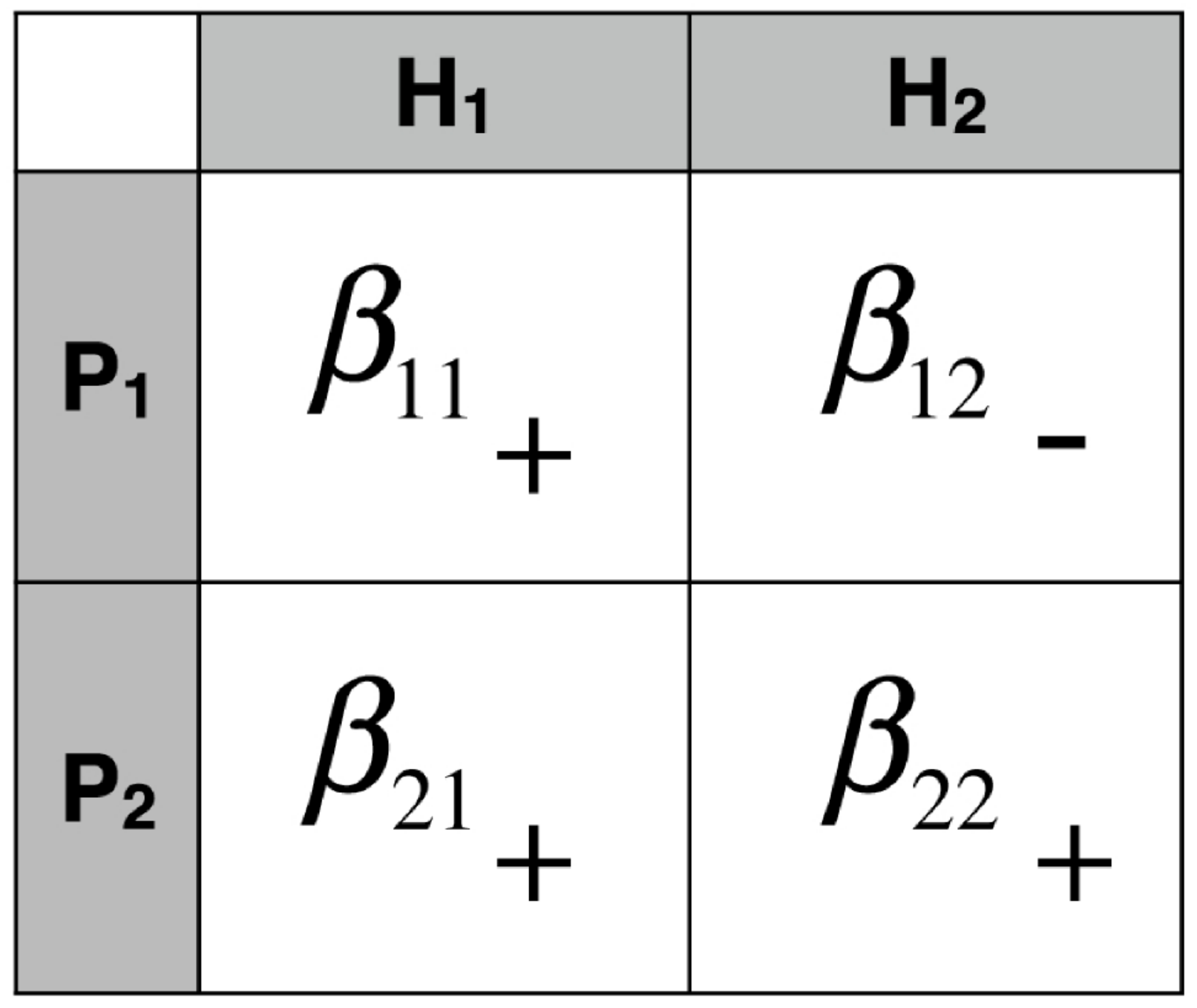

Figure 2 


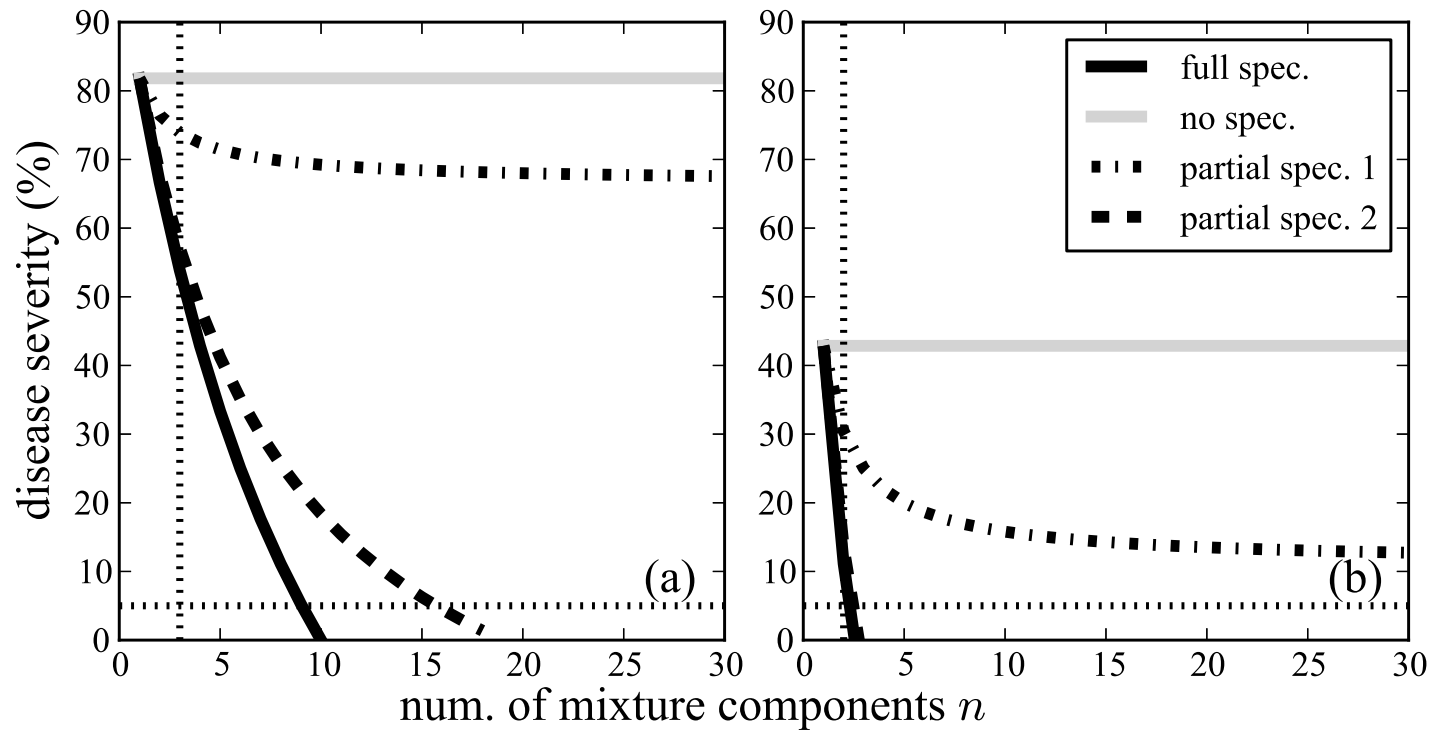

Figure 3 

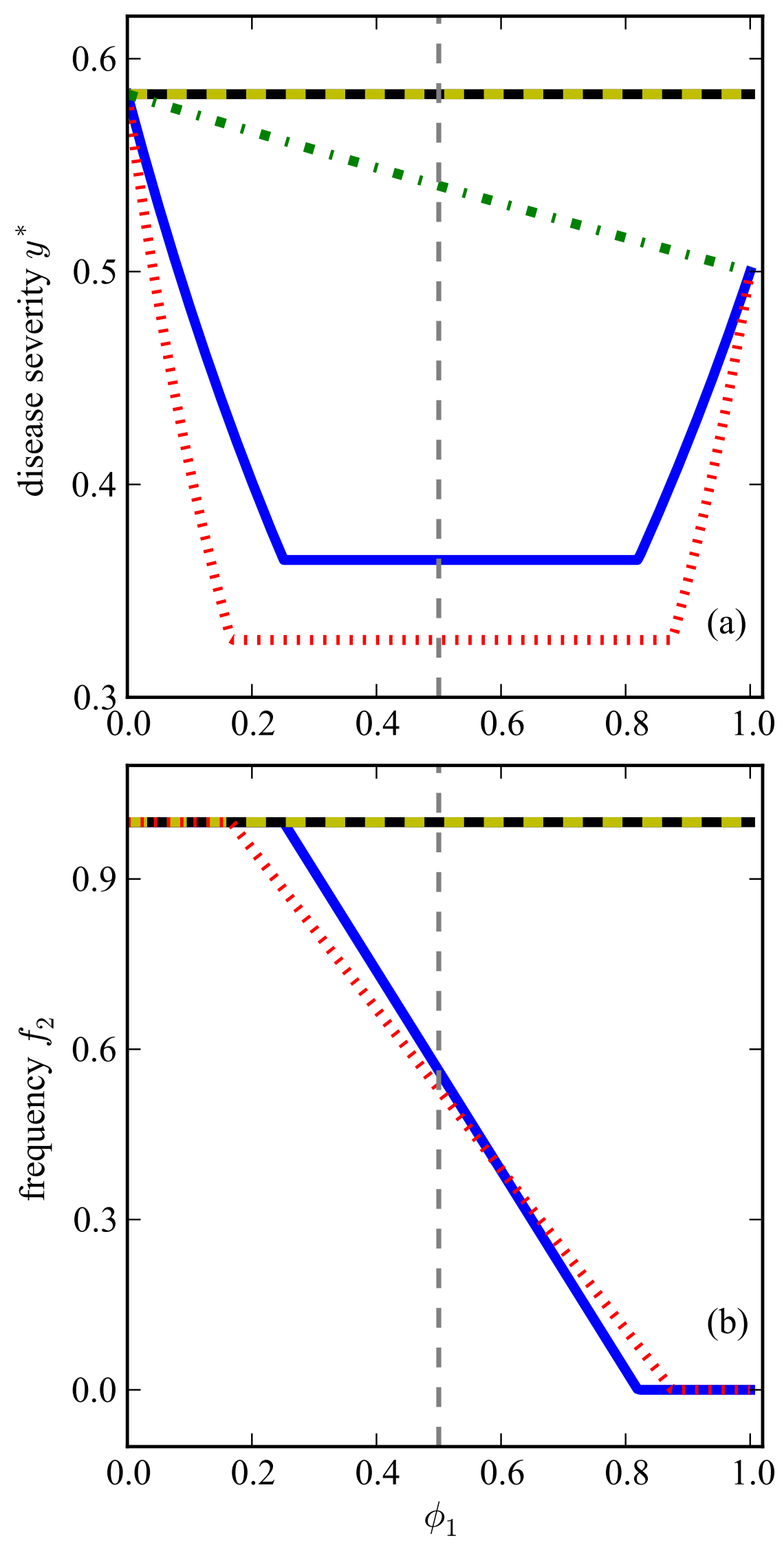

Figure 4 